\title{
Artificial Neural Network Application for Aroma Monitoring on The Coffe Beans Blending Process
}

\author{
Roza Susanti \#, Zas Ressy Aidha \#, Milda Yuliza \#, Suryadi \#, Surfa Yondri \# \\ \# Department of Electrical Engineering, Politeknik Negeri Padang, Indonesia \\ E-mail: rozaaznir@gmail.com,zasressya@gmail.com,mildayuliza@yahoo.com,suryadi2708@yahoo.com,surfa_yondri@yahoo.com
}

\begin{abstract}
This study aims to identify the type of coffee powder aroma from the coffee beans blending using backpropagation artificial neural network (ANN). Backpropagation is a controlled training implementing a weight adjustment pattern to achieve a minimum error value between the the predicted and the actual output. Within this study, the coffee aroma testing utilized electronic tasting sensor system consisted of 4 sensors namely TGS 2611, TGS 2620, TGS 2610 and TGS 2602 . The coffee aroma monitoring and data collection in this system applied LabVIEW software as a virtual instrumentation. The testing result of this ANN was able to distinguish the coffee variety of Robusta, Arabica coffee powder and the one without any coffee aroma. The backpropagation architecture was formed by 3 layers consisting of 1 input layer with 4 input nerve cells, 1 hidden layer with 8 neural cells, and 2 output layers by applying the backpropagation training algorithm. The training data was taken from 70 data samples of each circumstance of coffee with 5 testing times. The results of the training and testing showed that the established backpropagation was capable to identify and differenciate the coffee powder in accordance with the given input with different average success rate; $\mathbf{9 1 . 9 6 \%}$ for Robusta coffee, $100 \%$ for Arabica coffee, and no $\mathbf{8 4 . 2 4 \%}$ for without coffee aroma.
\end{abstract}

Keywords - Coffee powder, Aroma, Electronic Tasting, Gas Sensors, LabVIEW and Backpropagation.

\section{INTRODUCTION}

Indonesia is one of the largest coffee producer and exporter countries in the world [1]. In 2016, Indonesia became the fourth major country producing coffee in the world after Brazil, Vietnam and Colombia. These four countries produce $69.25 \%$ of world coffee production. Indonesian and Vietnamese coffee production are still dominant in Robusta coffee [2], on the other hand, Brazil and Colombia are more dominant producing Arabica coffee. Coffee plantations in Indonesia mostly can be found on the southeern part of the equator, such as in Southern Sumatra, Lampung, Bengkulu, Java, Southern Sulawesi, Bali and Nusa Tenggara [1]. Generally, the coffee characteristics can be seen from its bean shape, size, color, taste and aroma. To determine its aroma, coffee farmers usually smell the aroma right on the bean or on the coffee powder to find out the variety and quality of its aroma. It is a traditional way and totally depends on the sense of human taste. Usually, this aroma testing depending upon the human taste is unstable, because it relies on the taster physical or mental condition within the process. Therefore, it is only qualitative measures that can be determined. To perceive the coffee aroma, the system of electronic tasting sensor or electronic nose that can produce objective measurement and user friendly is taken. This electronic nose is developed from a system that automatically detects and classifies odor, steam and gas [3].

This research was conducted in order to identify the coffee powder aroma using some gas sensor and data analysis applying backpropagation artificial neural network (ANN) method. The aim of using several gas sensors was to get the precise and maximum coffee flavor from the coffee beans blending process. Monitoring and sensor data retrieving were done with LabVIEW software as virtual instrumentation whereas for the identification process utilized Matlab software to identify coffee aroma by applying backpropagation principle.

\section{THE MATERIAL AND METHOD}

Coffee is a kind of tree that is widely planted in Asia, Latin America and Africa. The result from the extracting process of coffee beans is used as beverage mixing ingredients, and is usually served with boiled water [4].

In the trading world, known some varieties of coffee, however only Robusta, Arabica and Liberika are the ones often cultivated. This classification is generally based on its species, except for Robusta. Regardless, Robusta is not a species as its descendant deriving from several coffee species particularly Coffea canephora [6]. 
The monitoring and control process were conducted using LabVIEW software which was considered as an integral part of virtual instrumentation as it provided an easy-to-use application development environment and was tailored to the needs of engineers and scientists. This software offers sophisticated features which is easily connected to a variety of other hardwares and softwares. Graphical programming is one of the most powerful features offered by LabVIEW to engineers and scientists. By utilizing this software, users could design custom virtual instrumentation by creating a graphical user interface on the computer screen, as listed below:

- Operating the instrumentation program

- Controlling hardware

- Analyzing data

- Displaying results

Users could create front panels with knobs, buttons, dials, and graphics to mimic traditional instrument control panels, to create custom testing panels, or to visually representing the operation and control of the process.

A sensor is a device for detecting / measuring something to convert mechanical, magnetic, heat, light beam and chemical variations becoming voltage and electric current. The sensor itself consists of a transducer with or without a signal amplifier / processor formed in a sensing system. In the controlling and robotics systems environment, sensor gives the eye-like, hearing, nose, and tongue-like similarities that will be processed by the controller as its brain. Accordingly, this research took the gas sensor type namely Taguchi Gas Sensor (TGS) to be applied.

In general, the ANN process is divided into 2 parts, namely training and testing. Training is a learning process of the neural network system regulating the input value and how the mapping on the output works until the appropriate model obtained. On the other hand, testing is the examining accuracy process of the obtained model from the training process. [8]. An example of a backpropagation network with one hidden layer can be seen in the following figure:

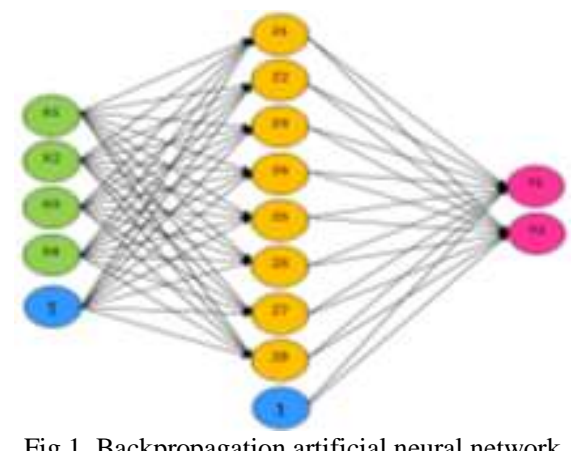

III. RESUlt AND DisCUSSION

In the circuit design, economic value of component use should be taken into account. Before building the circuit and the system, initially block diagram was planned. It was for one purpose of having circuit leading to the desired destination. As a controller, Microcontroller was taken, as seen in the blockdiagram illustrated in Figure.

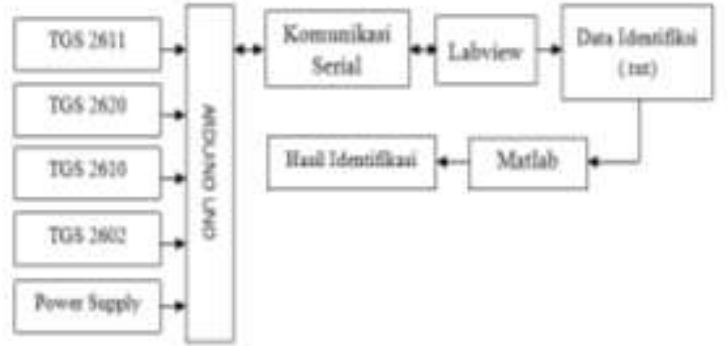

Fig 2. Block Diagram Process

The tool design was built by having some working principles. Initially, the coffee powder was put into the test container. Once the tool was activated, then the coffee powder in the test container would begin the stages of the process for the aroma identification. Four TGS sensors were installed inside the container which was useful for detecting the aroma. This sensor would continuously monitor the odor changes in the container. Afterward, monitored sensor data was stored into the user's PC in the form of .txt format. This stored data would be the input data for the identification process using artificial neural network in Matlab software. Learning outcomes would constantly be conducted until reaching the identification process, where the system was capable to detect the appropriate coffee odor based on the coffee input. When the identification result was in accordance with the desired target (Output $=$ Target), the identification process completed. This process would invariably repeat prior to the desired coffe variety perceived. The identification of the variety was referred to the level of aroma drifting up from the coffee powder and was detected by the TGS sensor.

TGS stands for Taguchi Gas Sensor which is a sensor that can detect odor in the environment around the sensor.

TGS has a sensor resistance where its value is depended on the presence of oxygen. When the presence of the oxygen on the tin oxide layer increases, the potential barrier level and the sensor resistance value increase as well. This TGS sensor has 4 foot pin where pin 3 and pin 4 are conjoined to VCC $5 \mathrm{~V}$, pin 1 to GND and pin 2 to resistor, and these pins are also recognized as output data.

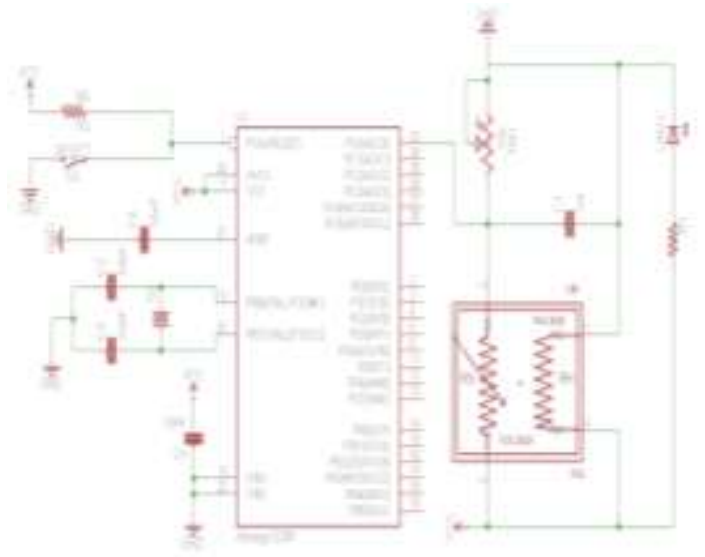

Fig 3. Electronic System Circuit

This research carried out a design in order to give brief overview to the users concerning the hardware system that would be built. In designing the hardware / mechanics, some mechanical parts were required forming a complex design. 
Those parts were funnel section, grinding container, sockets for the electronics, AC motor enclosures and final container of coffee powder. Seen in the picture below is the design of the going to be built mechanical design.

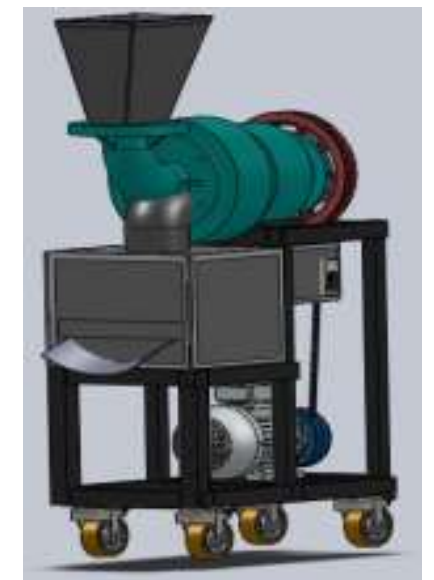

Fig 4. Mechanical Tool and Its Design

When all designing activities completed, it was continued to test the tools. This testing utilized Matlab software as identification, and was conducted in two stages, they were testing on the training process and on the identification process. It can be seen in sensor response graph as input data in the training process.

Seen in Figure 8 is the GUI training display when the entire sample data has been inputted with the temporary weight using the random weight. The next process was holding the input training with the desired target. Figure 9 views the conducted training process.

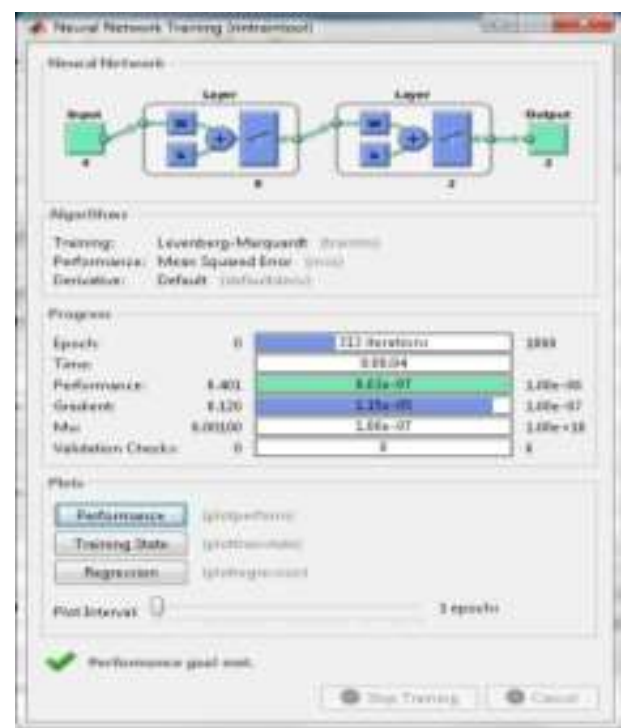

Fig 5. Display of artificial neural network training

Taken from the training process, graph of performance, training state and regression can be viewed.

From the graph above, it can be seen that error goal value (MSE) is 0.0096306 accomplished at the 313th epoch. Regression value is 1 showing the fit data between input data and target. Completing the training process, then the training result in the GUI was displayed in the form of training accuracy level, total data amount, the correct and incorrect data amount, as hown in Figure. 6 below.

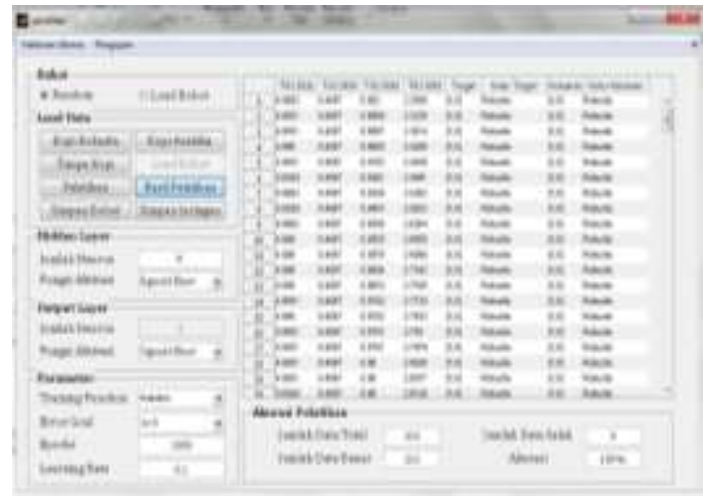

Fig 6. Display of training result

Identification as follows:

a. Identification of Robusta coffee powder

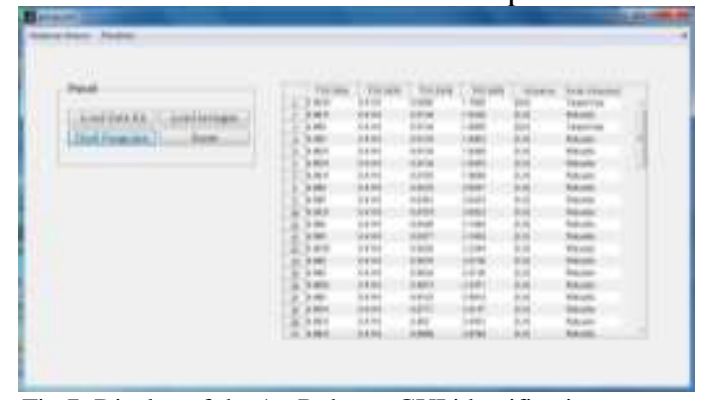

Fig 7. Display of the 1st Robusta GUI identification process

Following is the graph of TGS sensor response as input data in identification process.

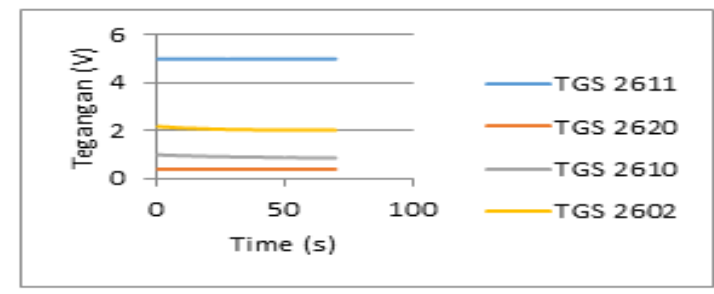

Fig 8. TGS sensor response on Robusta as input data on the 1st identification process

From the result of identification process on Robusta coffee powder above, it was in the same line with the input given. So the success rate of this program taken from the whole identification data was as below:

$$
\frac{69}{70} \times 100 \%=98,5 \%
$$

The success rate of the identification process above was $98.5 \%$.

Following is the graph of TGS sensor response as input data in identification process.

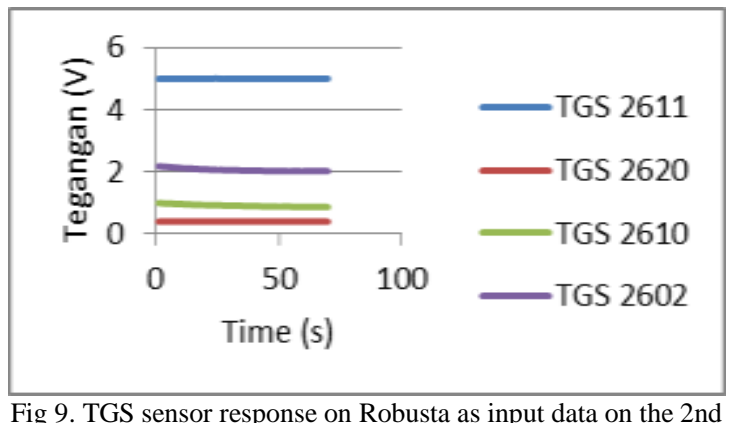

Fig 9. TGS sensor response on Robusta as input data on the 2nd identification process 
From the result of identification process on Robusta coffee powder above, it was in the same line with the input given. So the success rate of this program taken from the whole identification data was as below:

$$
\frac{63}{70} \times 100 \%=90 \%
$$

The success rate of the identification process above was $90 \%$.

Following is the graph of TGS sensor response as input data in identification process.

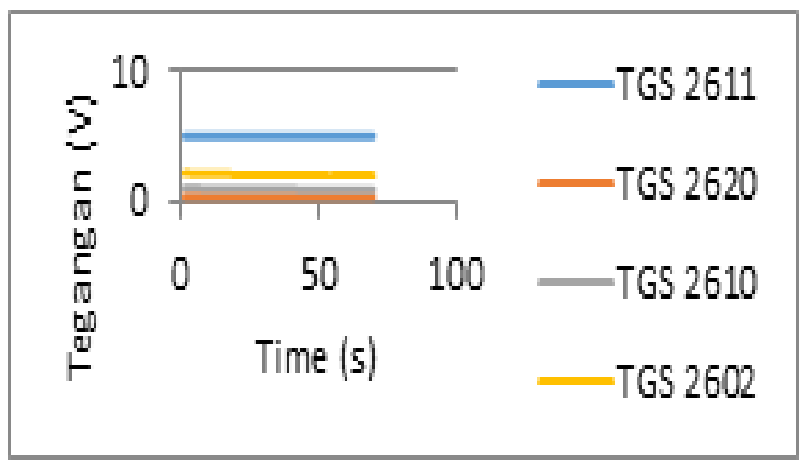

Fig 10. TGS sensor response on Robusta as input data on the 3 rd identification process

From the result of the identification process above, it was in the same line with the input given. So the success rate of this program taken from the whole identification data was as below:

$$
\frac{68}{70} \times 100 \%=97,1 \%
$$

The success rate of the identification process above was $97.1 \%$.

Following is the graph of TGS sensor response as input data in identification process.

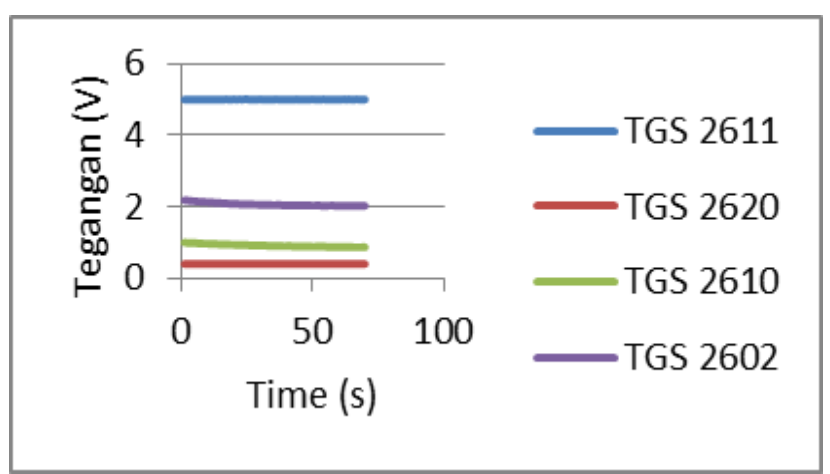

Fig11. TGS sensor response on Robusta as input data on the 4th identification process

From the result of identification process on Robusta coffee powder above, it was in the same line with the input given. So the success rate of this program taken from the whole identification data was as below:

$$
\frac{61}{70} \times 100 \%=87,1 \%
$$

The success rate of the identification process above was $87.1 \%$

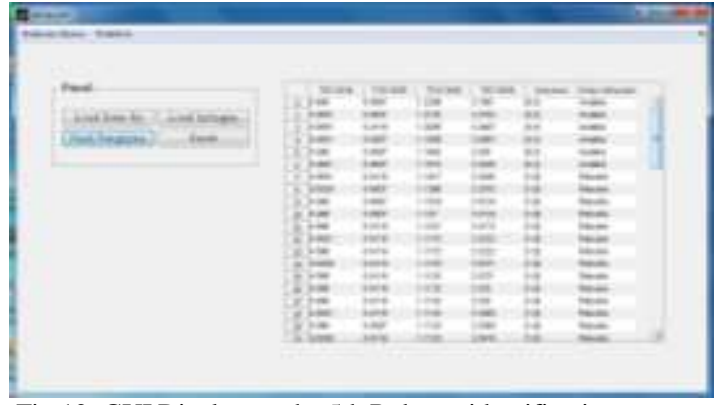

Fig 12. GUI Display on the 5th Robusta identification process

Following is the graph of TGS sensor response as input data in identification process.

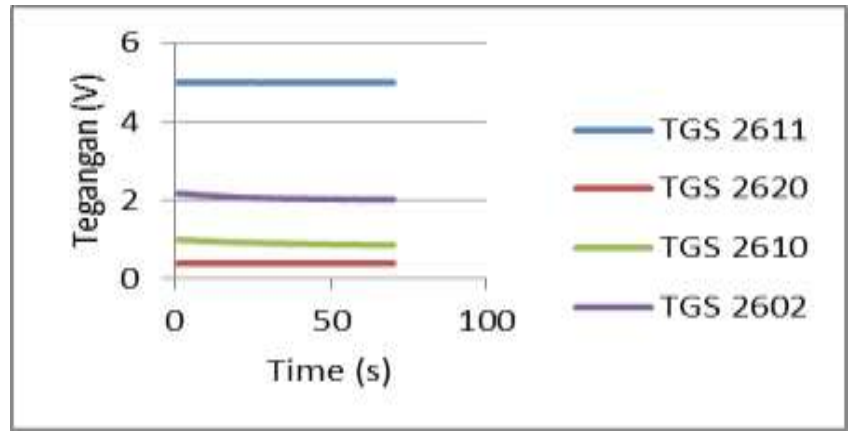

Fig 13. TGS sensor response on Robusta as input data on the 5th identification process

From the result of identification process on Robusta coffee powder above, it was in the same line with the input given. So the success rate of this program taken from the whole identification data was as below:

$$
\frac{64}{70} \times 100 \%=91,4 \%
$$

The success rate of the identification process above was $91.4 \%$

b. Identification without the coffee powder

Following is the graph of TGS sensor response as input data in identification process.

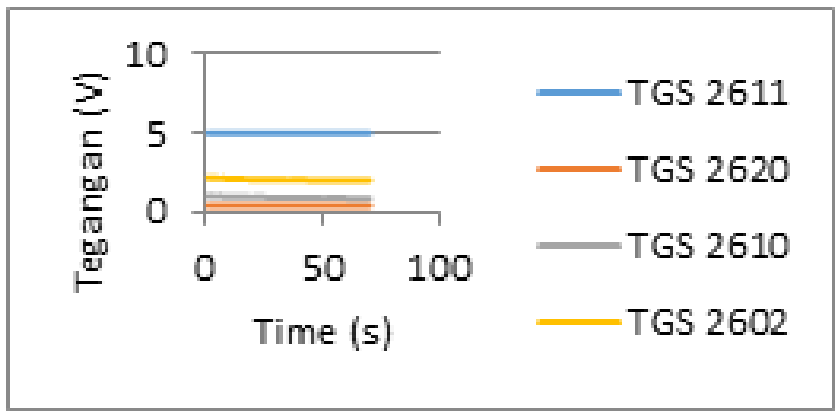

Fig 14. TGS sensor response without the coffee powder as input data on the 1 st identification process

From the result of identification process without the coffee powder above, it was in the same line with the input given. So the success rate of this program taken from the whole identification data was as below: 


$$
\frac{65}{70} \times 100 \%=92,8 \%
$$

The success rate of the identification process above was $92.8 \%$.

Following is the graph of TGS sensor response as input data in identification process.

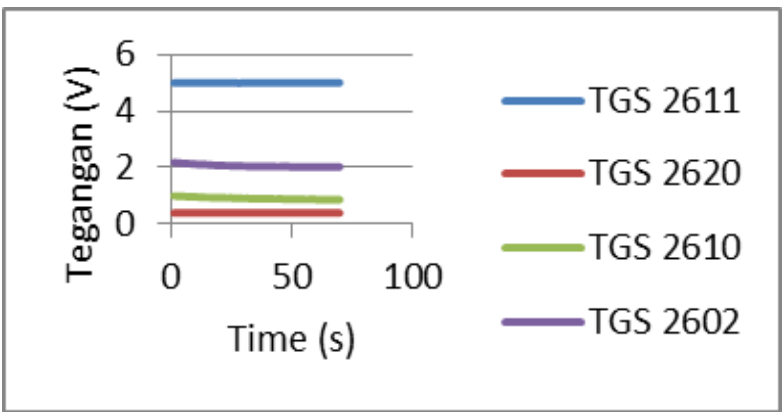

Fig 15. TGS sensor response without the coffee powder as input data on the 2nd identification process

From the result of identification process without the coffee powder above, it was in the same line with the input given. So the success rate of this program taken from the whole identification data was as below:

$$
\frac{69}{70} \times 100 \%=98,5 \%
$$

The success rate of the identification process above was $98.5 \%$.

Following is the graph of TGS sensor response as input data in identification process.

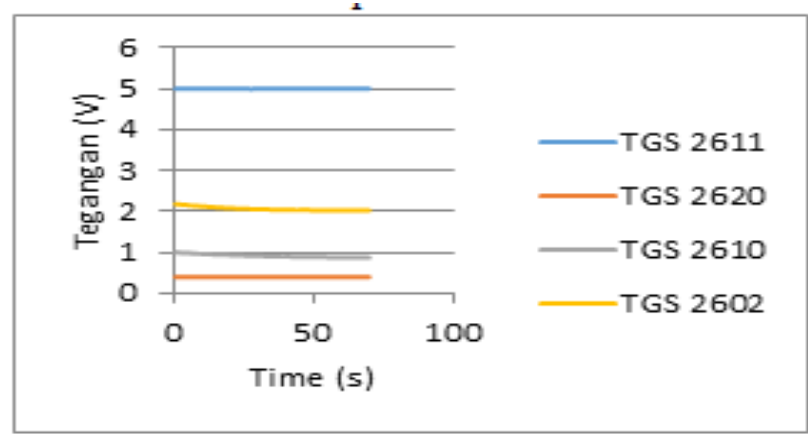

Fig 16. TGS sensor response without the coffee powder as input data on the 3rd identification process

From the result of identification process without the coffee powder above, it was in the same line with the input given. So the success rate of this program taken from the whole identification data was as below:

$$
\frac{53}{70} \times 100 \%=75,7 \%
$$

The success rate of the identification process above was $75.7 \%$

Following is the graph of TGS sensor response as input data in identification process.

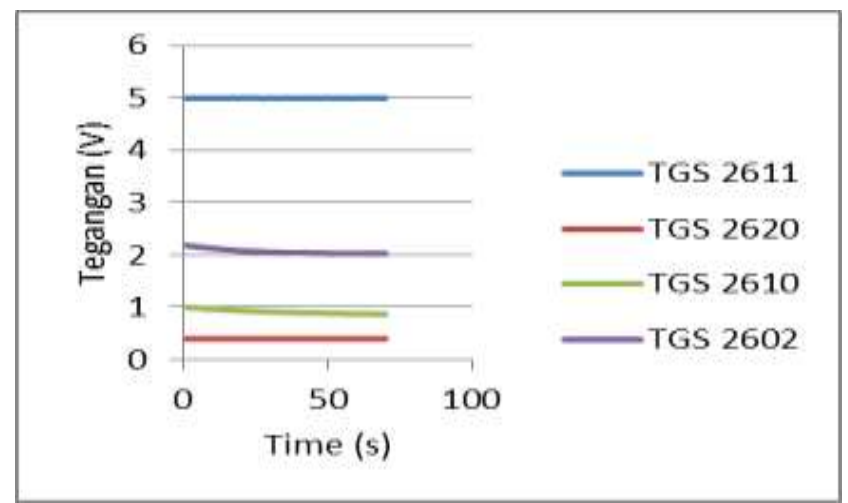

Fig 17. TGS sensor response without the coffee powder as input data on the 4 th identification process

From the result of identification process without the coffee powder above, it was in the same line with the input given. So the success rate of this program taken from the whole identification data was as below:

$$
\frac{54}{70} \times 100 \%=77,1 \%
$$

The success rate of the identification process above was $77.1 \%$

Following is the graph of TGS sensor response as input data in identification process.

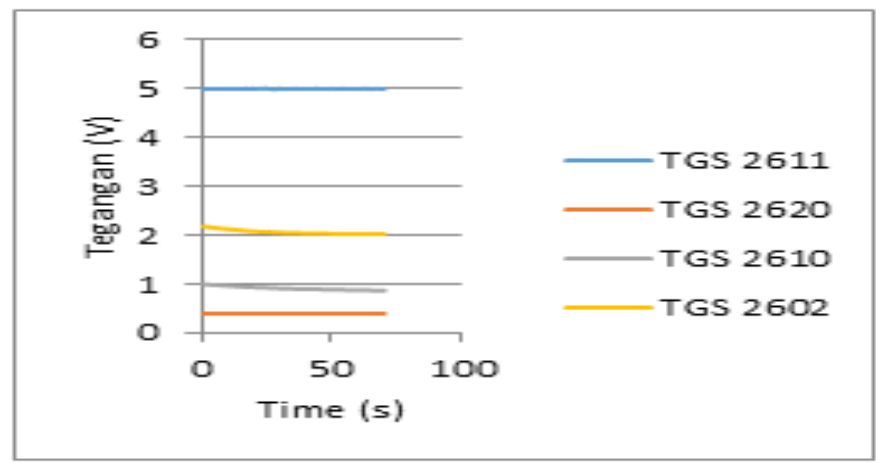

Fig 18. TGS sensor response without the coffee powder as input data on the 5 th identification process

From the result of identification process without the coffee powder above, it was in the same line with the input given. So the success rate of this program taken from the whole identification data was as below:

$$
\frac{54}{70} \times 100 \%=77,1 \%
$$

The success rate of the identification process above was $77.1 \%$

In the identification process of Robusta, Arabica coffee powder and the one without coffee powder using backpropagation artificial neural network, all data from each TGS sensor was the starting point data up to the stable point. It was due to at this point, the circumstances around the sensor has been occupied by the aroma of provided coffee powder . 
Response data of each training gas utilized backpropagation which had 4 input nodes, 8 hidden nodes and 2 output nodes. This research took 210 data for the training process consisting of 70 data each on Robusta, Arabica coffee powder aroma and the one without coffee powder. Each coffee aroma data was provided with a target value such as Robusta coffee powder with 10 as the target, Arabica with 01 , and the one without coffee powder with 0 0 as its target.

Nevertheless, the training process had consumed much time as the system design was required to fulfill the given target. To stop the program, two conditions were implemented where the first condition was by limiting the number of epoch usage, and the second one was by setting the MSE (Mean Square Error) value. In this training process, binary sigmoid activation function was taken aiming to set the output value in the range of 0 and 1 , and linear (identity) function was to set the equal value on both input and output $(\mathrm{y}=\mathrm{x})$. To accelerate the training process, LevenbergMarquardt (LM / trainlm) algorithm was applied which was one of the backpropagation functions of standard numerical optimization techniques. As a matter of the fact, the trainlm learning function is the fastest algorithm for large feed forward neural network training (up to hundreds of weight). After the training process was completed, the next weight value would be obtained in order to be used for the identification process.

This identification process was conducted totally 5 times on identification test with 70 sample data within 1 time test. To get the appropriate result with the given input, the number of error goal in this process put up insufficient value of 0.000001 with the objective to stop the identification process. It was done by limiting the insufficient error value. From the testing process that had been performed, the average of the identification success rate on 5 times testing with 3 conditions could be elaborated as follows:

a. Robusta Coffee Powder

$$
\text { Average success }=\frac{459,896}{5}=91,96 \%
$$

b. Without Coffee Powder

$$
\text { Average success }=\frac{421_{2} 236}{5}=84,24 \%
$$

\section{CONCLUSSION}

Based on the results of research and data analysis conducted in this study, it can be summarized as follows:
1. The TGS 2611 sensor, TGS 2620, TGS 2610 and TGS 2602 have high sensitivity to changes in the presented gas element in Robusta and Arabica coffee powder.

2. The TGS 2602 sensor has a high sensitivity and rapid response onto changes in the gas element of the Robusta and Arabica coffee powder aroma compared to the other three sensors. Regardless, the TGS sensor response onto air changes in the powder cup container is significantly good. It can be seen from the sensor response graph and the changes on resistance value (R_S) sensor onto the $V_{R L}$. value changes.

3. From 5 times of identification result onto 3 types of odor state yield the success rate with $91,96 \%$ average success rate for Robusta coffee, and $84,24 \%$ for the one without the aroma.

4. Backpropagation method successfully detects the aroma of inputted Robusta and Arabica coffee powder.

\section{ACKNOWLEDGMENT}

We would like to give our gratitude to Simblitabmas Dikti and P3M PNP for the grant provided in 2016 on this research. We also thank the institution, colleagues who have provided insight and expertise that greatly assisted the process of this research.

\section{REFERENCES}

[1] Indonesia Investments. 2015. Kopi, (Online), (http://www.indonesia investments.com/id/bisnis/komoditas/kopi/item186, diakses 5 November 2016)

[2] International Coffee Organization. 2017. Total production by exporting countries, (Online), (http://www.ico.org/trade_statistics.asp section $=$ Statistics, diakses 3 April 2017)

[3] Fitra, Neni Olya. 2016. identification of coffee grounds using artificial neural network based on backpropagation, (Online), (https://id.scribd.com/ document/328221232/Bab-I-pendahuluan, diakses 5 November 2016)

[4] Department of Education and Culture. 1988. Big Indonesian Dictionary. Jakarta: Balai Pustaka

[5] ndonesian Industry Standard. 1972. Quality Standard of Powdered Coffee. National Standards Agency, Jakarta

[6] Najiyati, S., and Danarti. 1997. Coffee Cultivation and Post-Harvest Processing. Self-Helping Spreader, Jakarta

[7] Hendrick, et al. 2012. Characterization of Coffee Aroma Using Short Time Fourier Transform, (Online), (https://www.academia.edu/10358

Menggunakan_Short_Time_Fourier_Transform, diakses 5 November 2016)

[8] Siang, Jong Jek. 2005. Jaringan Syaraf Tiruan dan Pemrogramannya Menggunakan Matlab, Yogyakarta: Andi Offset 